\title{
3-D Printing Technique to Aid Surgical Planning in Treatment of Floating Shoulder Injury: A Case Report
}

Xinghuo $\mathrm{Wu}^{1}$, Rong Liư ${ }^{2}$, Yin An ${ }^{1}$, Liu Ouyang ${ }^{1}$, Shuhua Yang1, Cao Yang ${ }^{1}$ and Zhewei Ye ${ }^{1 *}$

${ }^{1}$ Department of Orthopaedic Surgery, Union Hospital, Tongji Medical College, Huazhong University of Science and Technology, Wuhan, Hubei, China ${ }^{2}$ Department of Orthopaedic Surgery, Puren Hospital of Whan, Wuhan University of Science and Technology, Wuhan, Hubei, China

\section{Abstract}

Background: According to the published literature, Ipsilateral displaced clavicle and scapular neck fractures, also called floating shoulder injury (FSI), can be managed conservatively or surgically; however, the therapeutic option for this rare injury remains controversial.

Case Description: We presented a 18-year-old male patient who suffered FSI of the right shoulder. A threedimensional (3D) impression of the injured shoulder joint was obtained to plan the operation. The patient was operated by open reduction and plate internal fixation (ORIF) of the fractures with the aid of 3-D printing model.

Literature Review: Our literature review included various treatments associated with FSI. We identified that no previous reports of floating shoulders treated by ORIF with the aid of 3-D printing technique.

Purposes and Clinical Relevance: This report illustrates the practicability of 3-D printing technique in surgical treatment, which could enhance the surgeon's understanding the trauma of FSI, resulting in increased surgery precision and decreased trauma.

\section{Introduction}

The term "floating shoulder" was introduced by Herscovici in 1992 [1], charactered by ipsilateral scapular neck and clavicular shaft fractures. The definition of 'floating shoulder" has been criticized by many authors, and the widely accepted definition is a double disruption of the superior suspensory shoulder complex (SSSC) [2-5]. SSSC is a bony/soft tissue structure, playing important roles in the stability and some mobility of the shoulder girdle. Therefore, floating shoulder injury (FSI) involves the double destroy of osseous structure and ligament complex as well. Generally, the injury is caused by a highenergy trauma directly onto the shoulder, and traffic accident is one of the most common cases. It's difficult to manage the trauma without a thorough understanding of the complex anatomy of the shoulder girdle. Current best practice methods are used to obtain a three-dimensional (3D) impression of a injured shoulder joint to plan the operation. Especially, the rapid development of three-dimensional (3D) printing technology has proven efficacy in the fabrication of a shoulder joint with excellent dimensional accuracy. The goal of the preliminary study was explore the feasibility and reality of the low cost $3 \mathrm{D}$ printed model in the treatment of FSI. We presented a 18-year-old male patient which suffered FSI. I believe this is the first reported case of a floating shoulder treated by ORIF with the aid of 3-D printing technique.

\section{Case Report}

This case report didn't need the approval of the investigational Review Board.

A 18-year-older male patient sustained a traffic accident on the right shoulder. He was initially seen at a local hospital after injury. An anteroposterior X-ray was performed and the patient was diagnosed as clavicle and scapular fractures by the emergency doctor. Then the patient was transferred to our department of orthopedics. Then a computed tomography (CT) scan of the right shoulder with 3-D reconstruction was done, which revealed a comminuted right calvicle and acromial fractures, combined with scapular neck fracture (Figure 1). Based on the patient's CT DICOM data, then, a 3D shoulder model was printed (Figure 2). Preoperatively, the 3D printing model of the injured shoulder was used to evaluate the size and shape of the defect,

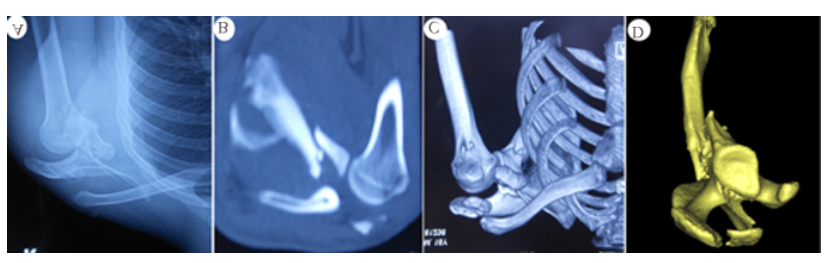

Figure 1: A patient with displaced, ipsilateral scapular neck and clavicular fractures. (A) A preoperative anteroposterior X-ray view of the right shoulder. (B-D) Preoperative computed tomography (CT) scans and 3D reconstruction showing a double disruption of the SSSC resulting in a floating shoulder.

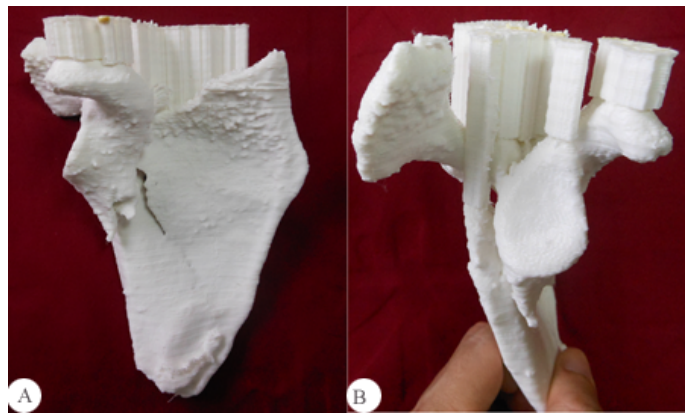

Figure 2: 3D printed model of the right shoulder. (A) Front view (B) side view.

*Corresponding author: Zhewei Ye, Department of Orthopaedics, Union Hospital, Tongji Medical College, Huazhong University of Science and Technology, Wuhan 430022, Hubei, China, Tel: +86 278754 1114; E-mail: 56138779@qq.com

Received September 15, 2015; Accepted November 16, 2015; Published November 25,2015

Citation: Wu X, Liu R, An Y, Ouyang L, Yang S, et al. (2015) 3-D Printing Technique to Aid Surgical Planning in Treatment of Floating Shoulder Injury: A Case Report. J Trauma Treat 4: 272. doi:10.4172/2167-1222.1000272

Copyright: (c) $2015 \mathrm{Wu} \mathrm{X}$, et al. This is an open-access article distributed under the terms of the Creative Commons Attribution License, which permits unrestricted use, distribution, and reproduction in any medium, provided the original author and source are credited. 


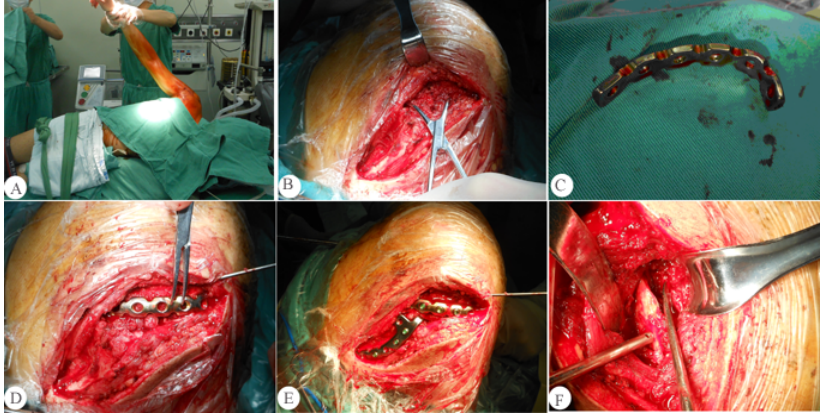

Figure 3: Intaoperative digital photograph demonstrating the operation process. (A) The patient was placed in left lateral position (B) open reduction of the displaced acromion $(C)$ the pre-bended plate(D) fixation the acromion with plate $(E)$ fixation the fractured clavicle with plate $(F)$ reduction of the displaced scapular neck.

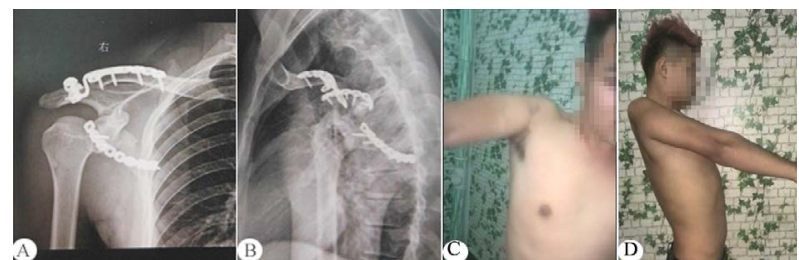

Figure 4: Postoperative imaging of the shoulder. Postoperative AP (A) and lateral radiographs $(B)$ of the right shoulder. Six months after operation, the degree of shoulder abduction (C) and shoulder anteflexion (D).

then to simulate the operation and make the operation plan. The main fractured fragments contained the clavicle and the scapular neck. The clavicle was intact, but the acromioclavicular joint was significantly displaced with the coracoid fragment. The right shoulder showed moderate soft tissue swelling, bruises, without neurovascular injury. The patient was subsequently operated by open reduction and internal fixation of the fracture. Open reduction and internal fixation (ORIF) was performed under general anaesthesia. The patient was placed on a radiolucent table in left lateral position. Firstly, the lateral calvicle and acromial area were surgically exposed. The acromion was open reduced and fixed with pre-bended reconstruction plate, followed by the reduction and fixation of the clavicle (Figure 3). The ligaments around the acromioclavicular joint were preserved and strengthened, including acromioclavicular capsular ligaments and coracoacromial ligament. Standard radiographs of the right shoulder were obtained the third day after operation, which confirmed an anatomically aligned of the acromioclavicular joint (Figure 4).

\section{Discussion}

The superior shoulder suspensory complex (SSSC), a bone and soft tissue structure, comprised of the glenoid, coracoid, and acromial processes, the CC ligament, the lateral clavicle, and the AC joint. Each of its components has its own individual function, together, maintain a normal, stable joint. Injuries to SSSC, also known as the floating shoulder, results in a adverse healing and long term functional consequences of the unstable shoulder $[3,6]$. Based on the concept of SSSC, a double disruption of the bone and soft tissue may be effectively to explain the unstable situation of the injured shoulder. The FSI consists of a double disruption of the scapula neck and clavicle fracture, associated with coracoacromial and acromioclavicular ligament disruption. The "floating shoulder injuries" (FSI) is a rare injury consisting of ipsilateral displaced clavicle and scapular neck fractures, usually associated with complications and mortality. The incidence of FSI ranged from
$0.10 \%$ to $0.15 \%$ [1]. Classification of the clavicular and scapular neck fracture is reliably made by an AP Radiograph. Clavicular fractures are usually classified by the anatomical area: the middle third fractures (Group I), the lateral third fractures (Group II ), and the medial third fractures (group III) [7]. Among them, middle third fractures are the most common injury in patients with a floating shoulder [8]. Scapular neck fractures are also separated in two anatomical groups: type A (anatomic neck fractures) and type B (surgical neck fractures), and type $\mathrm{B}$ are the common fractures in the injury of floating shoulder. Three-dimensional CT scan and reconstruction images may be more intuitively for demonstrating the fractures, especially exact interpretation of the amount of fracture displacement and angulation of the glenoid fragment [9]. Based on literature, three treatment options for floating shoulder have been mentioned: conservative treatment, operative treatment only for clavicle, and operative treatment for both the clavicular and scapular neck fractures. Conservative treatment has been reported with good functional outcomes by retrospective studies $[5,10]$. Good clinical results are also reported for operative treatment $[11,12]$. According to the published literature, FSI can be managed conservatively or surgically; however therapeutic option for this rare injury remains controversial $[5,11,13,14]$. With the gradually deepening understanding of the disease, FSI were considered inherently unstable required clavicle fixation alone or ORIF for both fractures. The order of fixation remains debatable: clavicle first or Scapular neck first? Initial fixation of the clavicle may benefit the indirect reduction of the glenoid segment. If the displacement was corrected, clavicle fixation alone will be enough for the treatment of the disease; otherwise, the fracture of the Scapular neck should be operated and fixed. Conversely, reduction and fixation of the displaced glenoid segment are more important, which should be carried out first, not the fracture of the clavicle. In addition, coracoacromial ligament (CAL) is the only ligament connecting the proximal (scapular body, spine and acromion) and distal (glenoid and coracoid process) fragments of the shoulder, therefore, which is an important stable structure for the scapular neck fractures [15]. During the operation, the CAL should be repaired and reconstructed.

In order to obtain optimal restoration of function in trauma surgery, an accurate anatomic understanding the injured structure is required. Based on computer-aided design (CAD) data, three-dimensional (3D) visualization model could be produced, which could precisely show the anatomical details of tissue. CAD technique has been widely used in manufacturing design, and which has been introduced in the medical applications, especially for implant design and preoperative planning [16]. From the 3D reconstruction model, surgeons could get intuitive and accurate information of the interesting area, which will be helpful for preoperative surgical planning, resulting in reduced operative time and increased operative outcomes $[17,18]$. 3D printing technique, a type of rapid prototyping (RP) process, which is faster, more convenient, less expensive, and more appropriate, compared to stereolithography. In recent years, 3D printing technique has been widely described and used in medical area, such as in skull deformities [19], mandibular deformities [20], facial deformities [21], and so on. The printed 3D model could enhance the surgeon's understanding of the morphology, and enable hands-on preoperative planning for the patient. Subsequent benefits may include increased surgery precision and decreased trauma.

\section{Conclusions}

3D printing model can intuitively reflect the pathological state of the patient, therefore, it could improve the operation accuracy and optimize the operation scheme for FSI. 
Citation: Wu X, Liu R, An Y, Ouyang L, Yang S, et al. (2015) 3-D Printing Technique to Aid Surgical Planning in Treatment of Floating Shoulder Injury: A Case Report. J Trauma Treat 4: 272. doi:10.4172/2167-1222.1000272

Page 3 of 3

\section{Acknowledgments}

Grant sponsor: National Natural Science Foundation of China. Grant Number: 81201393.

\section{References}

1. Herscovici D Jr, Fiennes AG, Allgöwer M, Rüedi TP (1992) The floating shoulder: ipsilateral clavicle and scapular neck fractures. J Bone Joint Surg $\mathrm{Br}$ 74: 362-364.

2. Ganz R, Noesberger B (1975) [Treatment of scapular fractures]. Hefte Unfallheilkd: 59-62.

3. Goss TP (1993) Double disruptions of the superior shoulder suspensory complex. J Orthop Trauma 7: 99-106.

4. Kumar VP, Satku K (1993) Fractures of clavicle and scapular neck. J Bone Joint Surg $\mathrm{Br}$ 75: 509 .

5. Ramos L, Mencía R, Alonso A, Ferrández $L$ (1997) Conservative treatment of ipsilateral fractures of the scapula and clavicle. J Trauma 42: 239-242.

6. Oh W, Jeon IH, Kyung S, Park C, Kim T, et al. (2002) The treatment of double disruption of the superior shoulder suspensory complex. Int Orthop 26: 145149

7. Allman FL Jr (1967) Fractures and ligamentous injuries of the clavicle and its articulation. J Bone Joint Surg Am 49: 774-784.

8. van Noort A, van der Werken C (2006) The floating shoulder. Injury 37: 218227

9. van Noort A, te Slaa RL, Marti RK, van der Werken C (2001) The floating shoulder. A multicentre study. J Bone Joint Surg Br 83: 795-798.

10. Labler L, Platz A, Weishaupt D, Trentz O (2004) Clinical and functional results after floating shoulder injuries. J Trauma 57: 595-602.

11. Hardegger FH, Simpson LA, Weber BG (1984) The operative treatment of scapular fractures. J Bone Joint Surg Br 66: 725-731.
12. Leung KS, Lam TP (1993) Open reduction and internal fixation of ipsilateral fractures of the scapular neck and clavicle. J Bone Joint Surg Am 75: 1015-1018.

13. Webber MC, Haines JF (2000) The treatment of lateral clavicle fractures. Injury 31: $175-179$.

14. Pailhes RG, Bonnevialle N, Laffosse J, Tricoire J, Cavaignac E, et al. (2013) Floating shoulders: Clinical and radiographic analysis at a mean follow-up of 11 years. Int J Shoulder Surg 7: 59-64.

15. Williams GR Jr, Naranja J, Klimkiewicz J, Karduna A, lannotti JP, et al. (2001) The floating shoulder: a biomechanical basis for classification and management. J Bone Joint Surg Am 83-83A: 1182-7.

16. Cohen A, Laviv A, Berman P, Nashef R, Abu-Tair J (2009) Mandibular reconstruction using stereolithographic 3-dimensional printing modeling technology. Oral Surg Oral Med Oral Pathol Oral Radiol Endod 108: 661-666.

17. Rozen WM, Ashton MW, Stella DL, Phillips TJ, Grinsell D, et al. (2008) The accuracy of computed tomographic angiography for mapping the perforators of the deep inferior epigastric artery: a blinded, prospective cohort study. Plast Reconstr Surg 122: 1003-1009.

18. Rozen WM, Ting JW, Baillieu C, Leong J (2012) Stereolithographic modeling of the deep circumflex iliac artery and its vascular branching: a further advance in computed tomography-guided flap planning. Plast Reconstr Surg 130 $380 \mathrm{e}-382 \mathrm{e}$.

19. Ono I, Abe K, Shiotani S, Hirayama Y (2000) Producing a full-scale model from computed tomographic data with the rapid prototyping technique using the binder jet method: a comparison with the laser lithography method using a dry skull. J Craniofac Surg 11: 527-537.

20. Chen Y, Niu F, Yu B, Liu J, Wang M, et al. (2014) Three-dimensional preoperative design of distraction osteogenesis for hemifacial microsomia. J Craniofac Surg 25: 184-188.

21. Cao D, Yu Z, Chai G, Liu J, Mu X (2010) Application of EH compound artificial bone material combined with computerized three-dimensional reconstruction in craniomaxillofacial surgery. J Craniofac Surg 21: 440-443. 